\title{
AN ALGORITHM FOR EXTRACTING THE GEOMETRIC PARAMETERS OF A RIGHT CIRCULAR CYLINDER FROM ITS ALGEBRAIC PARAMETERS
}

\author{
Mohamed Ali Said \\ Eng. Math. and Phys. Dept., Faculty of Engineering, Zagazig University, Egypt \\ E-mail: mmamsaid@hotmail.com
}

\begin{abstract}
The right circular cylinder (RCC) is an important geometric primitive that appears in many applications. The coefficients of the RCC's algebraic equation are called algebraic parameters. The algebraic parameters have no direct geometric meaning. The geometric parameters of RCC are a vector giving the direction of its axis, a point to fix the axis position, and a positive real number giving the radius of the cylinder. In this paper, an algorithm is introduced to extract the geometric parameters of a RCC from its algebraic parameters. The algorithm can also detect non RCC cases. Seven propositions are proved to make a solid theoretical ground for the algorithm. The algorithm is tested on five cases and produces exact results.
\end{abstract}

KEYWORDS: Algorithm, Right Circular Cylinder, Geometric Parameters, Algebraic Parameters, Quadrics.

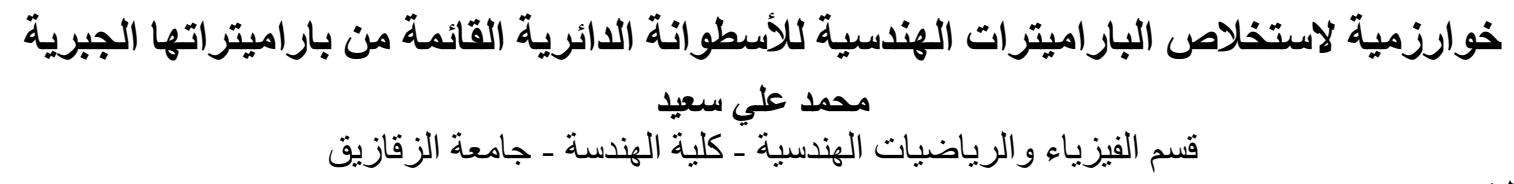

\section{INTRODUCTION}

A right circular cylinder (RCC) is one of the most important geometric primitives used in applications; a 95\% of industrial objects can be described by spheres, planes, cones, cylinders, and tori [Toony, Z. et al., 2015] and [Tran, T. et al., 2015].

The algebraic equation of a RCC as a quadric is given in [Ilyin, V.A. and Poznyak, E.G., 1984] as follows

$A x^{2}+B y^{2}+C z^{2}+D x y+E x z+F y z+G x+H y+I z+J=0$

Where the coefficients $A, B, \ldots J \in \mathbf{R}$, called the algebraic parameters, have little direct insight to the geometry of the surface. The conditions, under which equation (1.1) represents a 
RCC, is given in [Mortenson, M.E., 1990]. The RCC is described by a set of parameters called the geometric parameters. These parameters are: a vector $\lambda_{n} \mu_{n} v$ giving the direction of its axis, a point $\left(x_{0}, y_{0}, z_{0}\right)$ to fix the axis position, and a positive real number $R$ giving the radius of the cylinder. The geometric equation of a $\mathrm{RCC}$, in which the geometric parameters appear, is given in [Pogorelov, A.V., 1980] as:

$$
\left(x-x_{0}\right)^{2}+\left(y-y_{0}\right)^{2}+\left(z-z_{0}\right)^{2}-R^{2}=\frac{\left[\lambda\left(x-x_{0}\right)+\mu\left(y-y_{0}\right)+v\left(z-z_{0}\right)\right]^{2}}{\lambda^{2}+\mu^{2}+v^{2}}
$$

In this paper, an algorithm is introduced to extract the geometric parameters of a RCC from its algebraic parameters. Also, the algorithm can detect non RCC cases.

In literature, cylinders are subject of active research in many directions. Computing Cylinders from Minimal Sets of 3D Points [Beder, C. and Forstner, W., 2006], [Devillers, O. et al., 2001] and [Lichtblau, D., 2012]. Finding the Smallest Enclosing Cylinders to a set of data points [Watson, G.A., 2006], [Schomer, E. et al., 1996] and [Brandenberg, R. and Theobald, T., 2004]. Cylindrical objects detection, recognition and extraction [Tran, T. et al, 2015], [Figueiredo, R. et al., 2017], [Sarcar, M. et al., 2014], [Chaperon, T. and Goulette, F., 2001] and [Rabbani, T. and Heuvel, F., 2005]. Fitting of a cylinder to a set of data points [Lukacs, G. et al., 1998], [Al-Subaihi, I.A., 2016], [Nurunnabi, A. et al., 2017] and [Kwon, S. et al., 2003]. In all this work, the geometric parameters of a cylinder play a central role.

The remaining of the paper is arranged as follows: section 2 is devoted to comparing coefficients of the algebraic and the geometric equations, section 3 is devoted for extracting the components of the axis direction-vector, section 4 is devoted for extracting the coordinates of a point on the RCC's axis, in section 5 the radius of the RCC is extracted, in section 6 the proposed algorithm is introduced, section 7 is devoted for testing cases, and section 8 is devoted for conclusion. In the rest of this paper, cylinder means a right circular cylinder.

\section{BASIC RELATIONS BETWEEN PARAMETERS}

Expanding equation (1.2), the result is

$$
\begin{aligned}
& \left(\mu^{2}+v^{2}\right) x^{2}+\left(\lambda^{2}+v^{2}\right) y^{2}+\left(\lambda^{2}+\mu^{2}\right) z^{2}-2(\lambda \mu) x y-2(\lambda v) x z-2(\mu v) y z+ \\
& 2\left[\lambda\left(\mu y_{0}+v z_{0}\right)-\left(\mu^{2}+v^{2}\right) x_{0}\right] x+2\left[\mu\left(\lambda x_{0}+v z_{0}\right)-\left(\lambda^{2}+v^{2}\right) y_{0}\right] y+ \\
& \left.\left.2\left[v\left(\lambda x_{0}+\mu y_{0}\right)-\left(\lambda^{2}+\mu^{2}\right) z_{0}\right] z+\left(\mu^{2}+v^{2}\right) x_{0}^{2}+\lambda^{2}+v^{2}\right) y_{0}^{2}+\lambda^{2}+\mu^{2}\right) z_{0}^{2}- \\
& 2(\lambda \mu) x_{0} y_{0}-2(\lambda v) x_{0} z_{0}-2(\mu v) y_{0} z_{0}-\left(\lambda^{2}+\mu^{2}+v^{2}\right) R^{2}=0
\end{aligned}
$$

Comparing the coefficients of equation (1.1) and equation (2.1):

$$
\begin{aligned}
& A=\mu^{2}+v^{2}, \quad B=\lambda^{2}+v^{2}, \quad C=\lambda^{2}+\mu^{2} \\
& D=-2 \lambda \mu, \quad E=-2 \lambda v, \quad F=-2 \mu v \\
& G=-2\left(\mu^{2}+v^{2}\right) x_{0}+2 \lambda \mu y_{0}+2 \lambda v z_{0} \\
& \left.H=2 \mu \lambda x_{0}-2 \lambda^{2}+v^{2}\right) y_{0}+2 \mu v z_{0} \\
& \left.I=2 v \lambda x_{0}+2 v \mu y_{0}-2 \lambda^{2}+\mu^{2}\right) z_{0} \\
& \left.I=\left(\mu^{2}+v^{2}\right) x_{0}^{2}+\left(\lambda^{2}+v^{2}\right) y_{0}^{2}+C^{2}+\mu^{2}\right) z_{0}^{2}-2 \lambda \mu x_{0} y_{0}-2 \lambda v x_{0} z_{0}-2 \mu v y_{0} z_{0}-\left(\lambda^{2}+\mu^{2}+v^{2}\right) R^{2}
\end{aligned}
$$

\section{EXTRACTING THE AXIS DIRECTION-VECTOR}

In propositions (3.1) and (3.2), the cases in which the RCC's axis is parallel to one of the principal axes, are analysed. 


\section{Proposition (3.1):}

For the $R C C A x^{2}+B y^{2}+C z^{2}+D x y+E x z+F y z+G x+H y+I z+J=0$ with axis direction $\left(\lambda_{r} \mu_{r} v\right)$, if $D=E=F=0$ then the cylinder axis is parallel to one of the principal axes.

Proof: Substitute $D=E=F=0$ in equations (2.3) then

$$
\lambda \mu=0, \quad \lambda v=0, \quad \mu v=0
$$

and since $\lambda_{r} \mu, v$ can't be all zeros simultaneously, then the solution of system (3.1) is one of the following cases:

case 1: $\lambda \neq 0, \quad \mu=0, \quad v=0 \Rightarrow$ the cylinder axis is parallel to the $\mathrm{x}$-axis,

case 2: $\lambda=0, \quad \mu \neq 0, \quad v=0 \Rightarrow$ the cylinder axis is parallel to the y-axis, case 3: $\lambda=0, \quad \mu=0, \quad v \neq 0 \Rightarrow$ the cylinder axis is parallel to the $\mathrm{z}$-axis

\section{Proposition (3.2):}

For the RCC $A x^{2}+B y^{2}+C z^{2}+D x y+E x z+F y z+G x+H y+I z+J=0$ with axis direction $\left(\lambda_{r} \mu_{r} v\right)$, let $D=E=F=0$ then:

Case 1: If $A=0$ and $B=C>0$ then $\lambda=\sqrt{B}=\sqrt{C}, \mu=0, v=0$.

Case 2: If $B=0$ and $A=C>0$ then $\lambda=0, \mu=\sqrt{A}=\sqrt{C}, v=0$.

Case 3: If $C=0$ and $A=B>0$ then $\lambda=0, \mu=0, v=\sqrt{A}=\sqrt{B}$.

Note 1: If the conditions in case 1, case 2, or case 3 are not satisfied then multiply the cylinder's equation by (-1) and reconsider.

Note 2: The positive sign of the roots is taken but the negative sign can equally be used.

\section{Proof:}

Case 1: The positivity of $\mathrm{B}$ and $\mathrm{C}$ are direct consequence of equation (2.2).

Substitute $A=0$ and $B=C$ in equations (2.2) then

$$
\left.\begin{array}{c}
\mu^{2}+v^{2}=0 \\
\lambda^{2}+v^{2}=\lambda^{2}+\mu^{2}\left(\mu^{2}-v^{2}=0\right.
\end{array}\right\} \Rightarrow \mu=v=0
$$

Substitute from equation (3.2) in equations (2.2) then $\lambda=\sqrt{B}=\sqrt{C}$.

Case 2 and case 3 can be proved by a similar manner

The cases, where the RCC's axis is parallel to one of the principal planes, are treated in propositions (3.3) and (3.4).

\section{Proposition (3.3):}

For the $R C C A x^{2}+B y^{2}+C z^{2}+D x y+E x z+F y z+G x+H y+I z+J=0$, if one of the coefficients $\mathrm{D}, \mathrm{E}$, and $\mathrm{F}$ is not equal to zero and the other two are identically zeros, then the RCC's axis is parallel to one of the principal planes.

\section{Proof:}

Case 1: $D \neq 0, E=F=0$.

Substitute in equations (2.3) for $D, E$, and $F$, then

$\lambda \mu \neq 0 \Rightarrow \lambda \neq 0$ and $\mu \neq 0$.

$\lambda v=0 \Rightarrow v=0$ since $\lambda \neq 0$.

$\mu v=0 \Rightarrow v=0$ since $\mu \neq 0$.

Thus $\lambda \neq 0, \quad \mu \neq 0, v=0 \Rightarrow$ the RCC's axis is parallel to the xy-plane.

Case 2 and 3 are proved similarly 


\section{Proposition (3.4):}

For the $R C C A x^{2}+B y^{2}+C z^{2}+D x y+E x z+F y z+G x+H y+I z+J=0$ with $A>0$, $B>0$, and $C>0$

Case 1: if $D \neq 0, E=0$, and $F=0$ then $\lambda=\sqrt{B}, \mu=-\operatorname{sign}(D) \sqrt{A}, v=0, C=A+B$, and $D^{2}=4 A B$.

Case 2: if $D=0, E \neq 0$, and $F=0$ then $\lambda=\sqrt{C}, \mu=0, v=-\operatorname{sign}(E) \sqrt{A}$,

$B=A+C$, and $E^{2}=4 A C$.

Case 3: if $D=0, E=0$, and $F \neq 0$ then $\lambda=0, \mu=\sqrt{C}, v=-\operatorname{sign}(F) \sqrt{B}$,

$A=B+C$, and $F^{2}=4 B C$.

Proof:

Case 1: proposition (3.3) case $1 \Rightarrow v=0$.

Substitute $v=0$ in equations $(2.2) \Rightarrow A=\mu^{2}, B=\lambda^{2}, C=\lambda^{2}+\mu^{2} \Rightarrow \lambda= \pm \sqrt{B}$, $\mu= \pm \sqrt{A}$, and $C=A+B$.

Using $D=-2 \lambda \mu$ to fix the signs and without loss of generality, let $\lambda=\sqrt{B}$ then $\mu=-\operatorname{sign}(D) \sqrt{A}$ and $D^{2}=4 A B$.

Case 2 and case 3 can be proved by a similar manner

The next two propositions are devoted for processing the case where the RCC's axis is in general positions.

\section{Proposition (3.5):}

For the $R C C A x^{2}+B y^{2}+C z^{2}+D x y+E x z+F y z+G x+H y+I z+J=0$ with axis direction $\left(\lambda_{,} \mu_{s} v\right)$, if $D E F \leq 0$ then

$$
\operatorname{sign}(\hat{)}=-\operatorname{sign}(F), \operatorname{sign}(\varphi)=-\operatorname{sign}(E) \text {, and } \operatorname{sign}(v)=-\operatorname{sign}(D) \text {. }
$$

\section{Proof:}

$D E F<0 \Rightarrow \operatorname{sign}(D) \operatorname{sign}(E) \operatorname{sign}(F)=-$

$D=-2 \lambda \mu \Rightarrow \operatorname{sign}(\lambda) \operatorname{sign}(\mu)=-\operatorname{sign}(D)$

$E=-2 \lambda \nu \Rightarrow \operatorname{sign}(\lambda) \operatorname{sign}(v)=-\operatorname{sign}(E)$

$F=-2 \mu \nu \Rightarrow \operatorname{sign}(\mu) \operatorname{sign}(v)=-\operatorname{sign}(F)$

Multiply equation (3.3) by $\operatorname{sign}(D) \Rightarrow$

$$
[\operatorname{sign}(D)]^{2} \operatorname{sign}(E) \operatorname{sign}(F)=-\operatorname{sign}(D) \Rightarrow
$$

$$
\operatorname{sign}(E) \operatorname{sign}(F)=-\operatorname{sign}(D)
$$

Similarly, it can be proved that:

$\operatorname{sign}(D) \operatorname{sign}(F)=-\operatorname{sign}(E)$

$\operatorname{sign}(D) \operatorname{sign}(E)=-\operatorname{sign}(F)$

Substitute from equation (3.7) in equation (3.4) $\Rightarrow$

$\operatorname{sign}(\lambda) \operatorname{sign}(\mu)=\operatorname{sign}(E) \operatorname{sign}(F)$ 
Substitute from equation (3.8) in equation (3.5) $\Rightarrow$

$\operatorname{sign}(\lambda) \operatorname{sign}(v)=\operatorname{sign}(D) \operatorname{sign}(F)$

Substitute from equation (3.9) in equation (3.6) $\Rightarrow$

$\operatorname{sign}(\mu) \operatorname{sign}(\mathrm{v})=\operatorname{sign}(D) \operatorname{sign}(E)$

equations (3.10 - 3.12) and equation (3.3) $\Rightarrow$

$\operatorname{sign}(\lambda)=-\operatorname{sign}(F), \operatorname{sign}(\mu)=-\operatorname{sign}(E), \operatorname{sign}(v)=-\operatorname{sign}(D)$

\section{Proposition (3.6):}

For the RCC $A x^{2}+B y^{2}+C z^{2}+D x y+E x z+F y z+G x+H y+I z+J=0$ with axis direction $\left(\lambda_{r} \mu_{r} v\right)$, if $D E F<0,(-A+B+C)>0,(A-B+C)>0$, and $(A+B-C)>0$ then the RCC's axis is in general position with $\lambda=-\operatorname{sign}(F) \sqrt{\frac{-A+B+C}{2}}, \mu=-\operatorname{sign}(E) \sqrt{\frac{A-B+C}{2}}$, and $v=-\operatorname{sign}(D) \sqrt{\frac{A+B-C}{2}}$.

Note: if the conditions in the proposition are not satisfied then multiply the RCC's equation by $(-1)$ and reconsider.

Proof: Solving equations (2.2) for $\lambda^{2}, \mu^{2}$, and $v^{2}$ then $\lambda^{2}=\frac{-A+B+C}{2}, \mu^{2}=\frac{A-B+C}{2}$, and $v^{2}=\frac{A+B-C}{2}$.

Using proposition (3.5) then

$\lambda=-\operatorname{sign}(F) \sqrt{\frac{-A+B+C}{2}}, \mu=-\operatorname{sign}(E) \sqrt{\frac{A-B+C}{2}}$, and $v=-\operatorname{sign}(D) \sqrt{\frac{A+B-C}{2}}$

\section{EXTRACTING A POINT ON THE RCC'S AXIS}

In this section, the coordinates $x_{\mathbf{n}}, y_{\mathbf{n}}$, and $z_{\mathbf{n}}$ of a point on the cylinder axis is determined. It is clear that there are infinity of points on the axis, any one of them can be used.

\section{Proposition (4.1):}

If $A x^{2}+B y^{2}+C z^{2}+D x y+E x z+F y z+G x+H y+I z+J=0$ is a RCC with axis direction $\left(\lambda_{2} \mu_{r} v\right)$, then $\lambda G+\mu H+v I=0$.

Proof: Using equations (2.4-2.6) for $G, H$, and $I$ respectively, then $\lambda G+\mu H+v I=\lambda\left[-2\left(\mu^{2}+v^{2}\right) x_{0}+2 \lambda \mu y_{0}+2 \lambda v z_{0}\right]+\mu\left[2 \mu \lambda x_{0}-2\left(\lambda^{2}+v^{2}\right) y_{0}+2 \mu \nu z_{0}\right]$ $+v\left[2 v \lambda x_{0}+2 v \mu y_{0}-2\left(\lambda^{2}+\mu^{2}\right) z_{0}\right]$, expand the right hand side and simplify, the result is obtained

Now, return to determining $x_{0}, y_{0}$, and $z_{0}$ keeping in mind the result of proposition (4.1), that is, $\lambda G+\mu H+v I=\mathbf{0}$.

Solving equations (2.4), (2.5), and (2.6) simultaneously for $x_{0}, y_{0}$, and $z_{0}$ using GaussJordan elimination: 


$$
\begin{aligned}
& {\left[\begin{array}{ccccc}
-\left(\mu^{2}+v^{2}\right) & \lambda \mu & \lambda \nu & \vdots & \frac{G}{2} \\
\lambda \mu & -\left(\lambda^{2}+v^{2}\right) & \mu \nu & \vdots & \frac{H}{2} \\
\lambda \nu & \mu v & -\left(\lambda^{2}+\mu^{2}\right) & \vdots & \frac{I}{2}
\end{array}\right] \approx}
\end{aligned}
$$

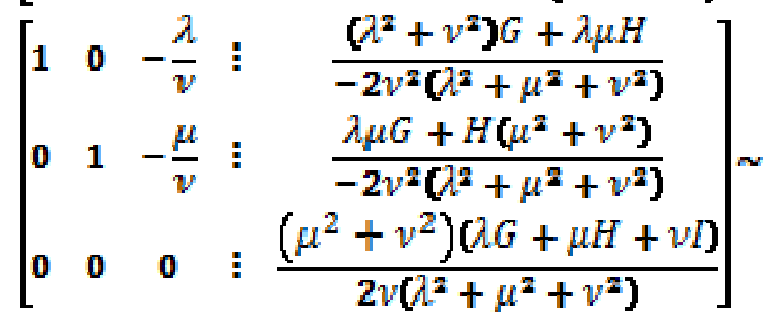

$$
\begin{aligned}
& {\left[\begin{array}{ccccc}
1 & 0 & -\frac{\lambda}{v} & \vdots & \frac{\left(\lambda^{2}+v^{2}\right) G+\lambda \mu H}{-2 v^{2}\left(\lambda^{2}+\mu^{2}+v^{2}\right)} \\
0 & 1 & -\frac{\mu}{v} & \vdots & \frac{\lambda \mu G+H\left(\mu^{2}+v^{2}\right)}{-2 v^{2}\left(\lambda^{2}+\mu^{2}+v^{2}\right)} \\
0 & 0 & 0 & 0 & 0
\end{array}\right]}
\end{aligned}
$$

Where $v \neq 0$.

The system has infinity of solutions as expected. The solutions are:

$x_{0}=\frac{\lambda}{v} z_{0}-\frac{\lambda \mu H+\left(\lambda^{2}+v^{2}\right) G}{2 \nu^{2}\left(\lambda^{2}+\mu^{2}+v^{2}\right)} \quad, \quad y_{0}=\frac{\mu}{v} z_{0}-\frac{\lambda \mu G+H\left(\mu^{2}+v^{2}\right)}{2 v^{2}\left(\lambda^{2}+\mu^{2}+v^{2}\right)}, \quad z_{0}=z_{0}$

Where $z_{0}$ is an arbitrary real number.

Similarly, with different pivoting, two different sets of solutions are obtained.

Under the condition $\lambda \neq 0$ the following set of solutions is obtained

$x_{0}=x_{0} \quad, \quad y_{0}=\frac{\mu}{\lambda} x_{0}-\frac{\mu \nu I+\left(\lambda^{2}+\mu^{2}\right) H}{2 \lambda^{2}\left(\lambda^{2}+\mu^{2}+v^{2}\right)}, \quad z_{0}=\frac{v}{\lambda} x_{0}-\frac{\mu v H+\left(\lambda^{2}+v^{2}\right) I}{2 \lambda^{2}\left(\lambda^{2}+\mu^{2}+v^{2}\right)}$

Where $x_{0}$ is an arbitrary real number.

and under the condition $\mu \neq 0$ the following set of solutions is obtained

$x_{0}=\frac{\lambda}{\mu} y_{0}-\frac{\lambda v I+\left(\lambda^{2}+\mu^{2}\right) G}{2 \mu^{2}\left(\lambda^{2}+\mu^{2}+v^{2}\right)} \quad, \quad y_{0}=y_{0}, \quad z_{0}=\frac{v}{\mu} y_{0}-\frac{\lambda v G+\left(\mu^{2}+v^{2}\right) I}{2 \mu^{2}\left(\lambda^{2}+\mu^{2}+v^{2}\right)}$

Where $y_{0}$ is an arbitrary real number.

The equations $(4.1-4.3)$ will be simplified by setting the arbitrary values to zeros and using $\lambda G+\mu H+\gamma I=0$ :

Case 1: $\lambda \neq \mathbf{0}$

$x_{0}=0, \quad y_{0}=\frac{\mu G-\lambda H}{2 \lambda\left(\lambda^{2}+\mu^{2}+v^{2}\right)}, \quad z_{0}=\frac{v G-\lambda I}{2 \lambda\left(\lambda^{2}+\mu^{2}+v^{2}\right)}$

Case 2: $\mu \neq 0$

$x_{0}=\frac{\lambda H-\mu G}{2 \mu\left(\lambda^{2}+\mu^{2}+v^{2}\right)}, \quad y_{0}=0, \quad z_{0}=\frac{v H-\mu I}{2 \mu\left(\lambda^{2}+\mu^{2}+v^{2}\right)}$

Case 3: $v \neq 0$

$$
x_{0}=\frac{\lambda I-v G}{2 v\left(\lambda^{2}+\mu^{2}+v^{2}\right)}, \quad y_{0}=\frac{\mu I-v H}{2 v\left(\lambda^{2}+\mu^{2}+v^{2}\right)}, \quad z_{0}=0
$$

\section{EXTRACTING THE RADIUS OF THE RIGHT CIRCULAR CYLINDER}

The radius $R$ will be obtained by solving equation (2.7) for $R^{2}$ : 
$R^{2}=\frac{\left(\mu^{2}+v^{2}\right) x_{0}^{2}+\left(\lambda^{2}+v^{2}\right) y_{0}^{2}+\left(\lambda^{2}+\mu^{2}\right) z_{0}^{2}-2 \lambda \mu x_{0} y_{0}-2 \lambda v x_{0} z_{0}-2 \mu v y_{0} z_{0}-J}{\lambda^{2}+\mu^{2}+v^{2}}$

Where $\lambda^{2}+\mu^{2}+v^{2} \neq 0$.

\section{THE PROPOSED ALGORITHM}

In the algorithm, the symbol " $\&$ " is used for the logical operator "AND".

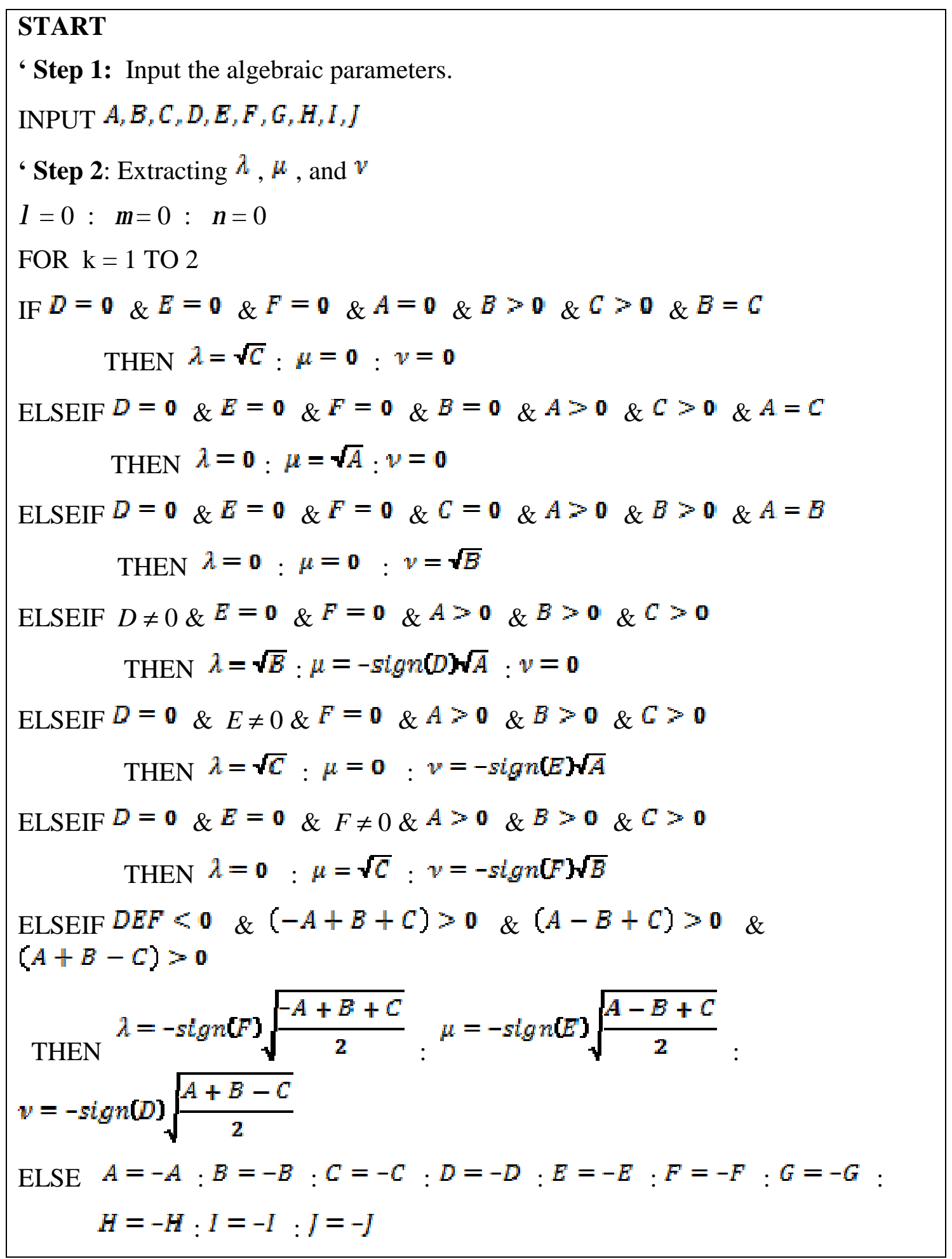




\section{ENDIF}

NEXT k

IF $\lambda=0 \& \quad \mu=0 \& v=0$ THEN OUTPUT “ Not a RCC” and STOP

' Step 3: Extracting $x_{\mathbf{n}}, y_{\mathbf{n}}$, and $z_{\mathbf{n}}$

$\mathrm{IF} \lambda G+\mu H+v I \neq 0$ THEN OUTPUT "Not a RCC" and STOP

IF $\lambda \neq 0 \quad$ THEN

$$
x_{0}=0 \quad: \quad y_{0}=\frac{\mu G-\lambda H}{2 \lambda\left(\lambda^{2}+\mu^{2}+v^{2}\right)} \quad: \quad z_{0}=\frac{v G-\lambda I}{2 \lambda\left(\lambda^{2}+\mu^{2}+v^{2}\right)}
$$

ELSEIF $\mu \neq 0$ THEN

$$
x_{0}=\frac{\lambda H-\mu G}{2 \mu\left(\lambda^{2}+\mu^{2}+v^{2}\right)} \quad: \quad y_{0}=0 \quad: \quad z_{0}=\frac{v H-\mu I}{2 \mu\left(\lambda^{2}+\mu^{2}+v^{2}\right)}
$$

ELSEIF $v \neq 0$ THEN

$$
x_{0}=\frac{\lambda I-v G}{2 v\left(\lambda^{2}+\mu^{2}+v^{2}\right)} \quad: \quad y_{0}=\frac{\mu I-v H}{2 v\left(\lambda^{2}+\mu^{2}+v^{2}\right)} \quad: \quad z_{0}=0
$$

ELSE OUTPUT "Not a RCC" and STOP

ENDIF

' Step 4: Extracting $R$

$\operatorname{IF}\left(\lambda^{2}+\mu^{2}+v^{2}\right) \neq 0 \quad \&$

$\left[\left(\mu^{2}+v^{2}\right) x_{0}^{2}+\left(\lambda^{2}+v^{2}\right) y_{0}^{2}+\left(\lambda^{2}+\mu^{2}\right) z_{0}^{2}-2 \lambda \mu x_{0} y_{0}-2 \lambda v x_{0} z_{0}-2 \mu v y_{0} z_{0}-\jmath>0\right.$

THEN

$R=\sqrt{\frac{\left(\mu^{2}+v^{2}\right) x_{0}^{2}+\left(\lambda^{2}+v^{2}\right) y_{0}^{2}+\left(\lambda^{2}+\mu^{2}\right) z_{0}^{2}-2 \lambda \mu x_{0} y_{0}-2 \lambda v x_{0} z_{0}-2 \mu v y_{0} z_{0}-J}{\lambda^{2}+\mu^{2}+v^{2}}}$

ELSE OUTPUT “Not a RCC” and STOP

ENDIF

' Step 5: Output the geometric parameters

OUTPUT $\lambda, \mu, v, x_{0}, y_{0}, z_{0}, R$

STOP

\section{TESTING CASES}

\subsection{Case 1}

$x^{2}+y^{2}-4=0 \Rightarrow$

$A=1, B=1, C=D=E=F=G=H=I=0, l=-4$.

$D=E=F=0, C=0, A>0, B>0, A=B \Rightarrow$ the axis is parallel to the $z$-axis and

$\lambda=0, \mu=0, v=\sqrt{B}=1$.

$\lambda G+\mu H+v I=0$ and $v=1 \neq 0 \Rightarrow$ 


$$
\begin{aligned}
& x_{0}=(\lambda I-v G) /\left[2 v\left(\lambda^{2}+\mu^{2}+v^{2}\right)\right]=0 \quad, \quad y_{0}=\frac{\mu I-v H}{2 v\left(\lambda^{2}+\mu^{2}+v^{2}\right)}=0 \quad, \quad z_{0}=0 \\
& \left(\lambda^{2}+\mu^{2}+v^{2}\right)=1 \neq 0 \text { and } \\
& {\left[\left(\mu^{2}+v^{2}\right) x_{0}^{2}+\left(\lambda^{2}+v^{2}\right) y_{0}^{2}+\left(\lambda^{2}+\mu^{2}\right) z_{a}^{2}-2 \lambda \mu x_{a} y_{a}-2 \lambda v x_{a} z_{a}-2 \mu v y_{a} z_{a}-l\right]=4>0 \Rightarrow} \\
& R=\sqrt{\frac{\left(\mu^{2}+v^{2}\right) x_{0}^{2}+\left(\lambda^{2}+v^{2}\right) y_{0}^{2}+\left(\lambda^{2}+\mu^{2}\right) z_{0}^{2}-2 \lambda \mu x_{0} y_{0}-2 \lambda v x_{0} z_{0}-2 \mu v y_{0} z_{0}-J}{\lambda^{2}+\mu^{2}+v^{2}}}=2 .
\end{aligned}
$$

See Figure (7.1).

\subsection{Case 2}

$$
\begin{aligned}
& 13 x^{2}+10 y^{2}+5 z^{2}-4 x y-6 x z-12 y z-56=0 \Rightarrow \\
& A=13, B=10, C=5, D=-4, E=-6, F=-12, G=H=I=0, I=-56 \\
& D E F=-288<0,(-A+B+C)=2>0,(A-B+C)=8>0, \text { and } \\
& (A+B-C)=18>0 \Rightarrow \text { the axis is in general position with } \\
& \lambda=-\operatorname{sign}(F) \sqrt{\frac{-A+B+C}{2}}=1, \mu=-\operatorname{sign}(E) \sqrt{\frac{A-B+C}{2}}=2, \text { and } \\
& v=-\operatorname{sign}(D) \sqrt{\frac{A+B-C}{2}}=3 .
\end{aligned}
$$

$\lambda G+\mu H+v I=0$ and $v=3 \neq 0 \Rightarrow$

$$
x_{0}=\frac{\lambda I-v G}{2 v\left(\lambda^{2}+\mu^{2}+v^{2}\right)}=0 \quad, \quad y_{0}=\frac{\mu I-v H}{2 v\left(\lambda^{2}+\mu^{2}+v^{2}\right)}=0 \quad, \quad z_{0}=0
$$

$\left(\lambda^{2}+\mu^{2}+v^{2}\right)=14 \neq 0$ and

$$
\begin{aligned}
& {\left[\left(\mu^{2}+v^{2}\right) x_{0}^{2}+\left(\lambda^{2}+v^{2}\right) y_{0}^{2}+\left(\lambda^{2}+\mu^{2}\right) z_{0}^{2}-2 \lambda \mu x_{0} y_{0}-2 \lambda v x_{0} z_{0}-2 \mu v y_{0} z_{0}-J\right]=56>0} \\
& R=\sqrt{\frac{\left(\mu^{2}+v^{2}\right) x_{0}^{2}+\left(\lambda^{2}+v^{2}\right) y_{0}^{2}+\left(\lambda^{2}+\mu^{2}\right] z_{0}^{2}-2 \lambda \mu x_{0} y_{0}-2 \lambda v x_{0} z_{0}-2 \mu v y_{0} z_{0}-J}{\lambda^{2}+\mu^{2}+v^{2}}}=2
\end{aligned}
$$

See Figure (7.2).

\subsection{Case 3}

$$
\begin{aligned}
& 392 x^{2}+596 y^{2}+596 z^{2}-560 x y-560 x z-392 y z+6048 x-5112 y-3528 z+15127=0 \\
& \Rightarrow A=392, B=596, C=596, D=-560, E=-560, F=-392, G=6048 \\
& H=-5112, I=-3528, I=15127 . \\
& D E F=-122931200<0,(-A+B+C)=800>0,(A-B+C)=392>0, \text { and } \\
& (A+B-C)=392>0 \Rightarrow \text { the axis is in general position with }
\end{aligned}
$$$$
\lambda=-\operatorname{sign}(F) \sqrt{\frac{-A+B+C}{2}}=20 \quad \mu=-\operatorname{sign}(E) \sqrt{\frac{A-B+C}{2}}=14 \text {, and }
$$$$
v=-\operatorname{sign}(D) \sqrt{\frac{A+B-C}{2}}=14 \text {. }
$$ 
$\lambda G+\mu H+v I=0$ and $\lambda=20 \neq 0 \Rightarrow$

$\left(\lambda^{2}+\mu^{2}+v^{2}\right)=792 \neq 0$ and

$$
x_{0}=0 \quad, \quad y_{0}=\frac{\mu G-\lambda H}{2 \lambda\left(\lambda^{2}+\mu^{2}+v^{2}\right)}=5.9 \quad, \quad z_{0}=\frac{v G-\lambda I}{2 \lambda\left(\lambda^{2}+\mu^{2}+v^{2}\right)}=4.9
$$

$$
\begin{aligned}
& {\left[\left(\mu^{2}+v^{2}\right] x_{0}^{2}+\left(\lambda^{2}+v^{2}\right) y_{0}^{2}+\left(\lambda^{2}+\mu^{2}\right) z_{0}^{2}-2 \lambda \mu x_{0} y_{0}-2 \lambda v x_{0} z_{0}-2 \mu v y_{0} z_{0}-J\right]} \\
& R=\sqrt{\frac{\left(\mu^{2}+v^{2}\right) x_{0}^{2}+\left(\lambda^{2}+v^{2}\right) y_{0}^{2}+\left(\lambda^{2}+\mu^{2}\right] z_{0}^{2}-2 \lambda \mu x_{0} y_{0}-2 \lambda v x_{0} z_{0}-2 \mu v y_{0} z_{0}-J}{\lambda^{2}+\mu^{2}+v^{2}}} \approx 3.295
\end{aligned}
$$

See Figure (7.3).

\subsection{Case 4}

$x^{2}+y^{2}+z^{2}-1=0 \Rightarrow$

$A=B=C=1, D=E=F=G=H=I=0, J=-1$

$D=E=F=0$, and no one of $A, B$, or $C$ is equal to zero $\Rightarrow$ Not a cylinder.

\subsection{Case 5}

$x^{2}+y^{2}-z^{2}=0 \Rightarrow$

$A=1, B=1, C=-1, D=E=F=G=H=I=J=0$

$D=E=F=0$, and no one of $A, B$, or $C$ is equal to zero $\Rightarrow$ Not a cylinder.

\subsection{Summary of the test cases}

\begin{tabular}{|c|c|c|c|c|c|}
\hline $\begin{array}{l}\text { Case No. } \\
\text { Parameters }\end{array}$ & 1 & 2 & 3 & 4 & 5 \\
\hline$A$ & 1 & 13 & 392 & 1 & 1 \\
\hline$B$ & 1 & 10 & 596 & 1 & 1 \\
\hline$C$ & $\mathbf{0}$ & 5 & 596 & 1 & -1 \\
\hline$D$ & 0 & -4 & -560 & $\mathbf{0}$ & 0 \\
\hline$E$ & $\mathbf{0}$ & -6 & -560 & $\mathbf{0}$ & $\mathbf{0}$ \\
\hline$F$ & $\mathbf{0}$ & -12 & -392 & $\mathbf{0}$ & 0 \\
\hline$G$ & $\mathbf{0}$ & $\mathbf{0}$ & 6048 & $\mathbf{0}$ & $\mathbf{0}$ \\
\hline$H$ & $\mathbf{0}$ & $\mathbf{0}$ & -5112 & $\mathbf{0}$ & 0 \\
\hline$I$ & $\mathbf{0}$ & $\mathbf{0}$ & -3528 & $\mathbf{0}$ & $\mathbf{0}$ \\
\hline$I$ & -4 & -56 & 15127 & -1 & 0 \\
\hline$\lambda$ & $\mathbf{0}$ & 1 & 20 & \multirow{7}{*}{ 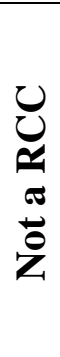 } & \multirow{7}{*}{ 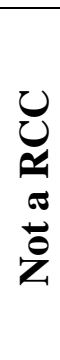 } \\
\hline$\mu$ & $\mathbf{0}$ & 2 & 14 & & \\
\hline$v$ & 1 & 3 & 14 & & \\
\hline$x_{0}$ & 0 & $\mathbf{0}$ & $\mathbf{0}$ & & \\
\hline$y_{0}$ & $\mathbf{0}$ & $\mathbf{0}$ & 5.9 & & \\
\hline$z_{0}$ & $\mathbf{0}$ & $\mathbf{0}$ & 4.9 & & \\
\hline$R$ & 2 & 2 & 3.295 & & \\
\hline
\end{tabular}

The test cases are summarized in Table (7.1).

Table (7.1): Summary of the Test Cases 


\subsection{Demonstration Figures for some of the test cases.}

The following figures demonstrate the first three test cases.

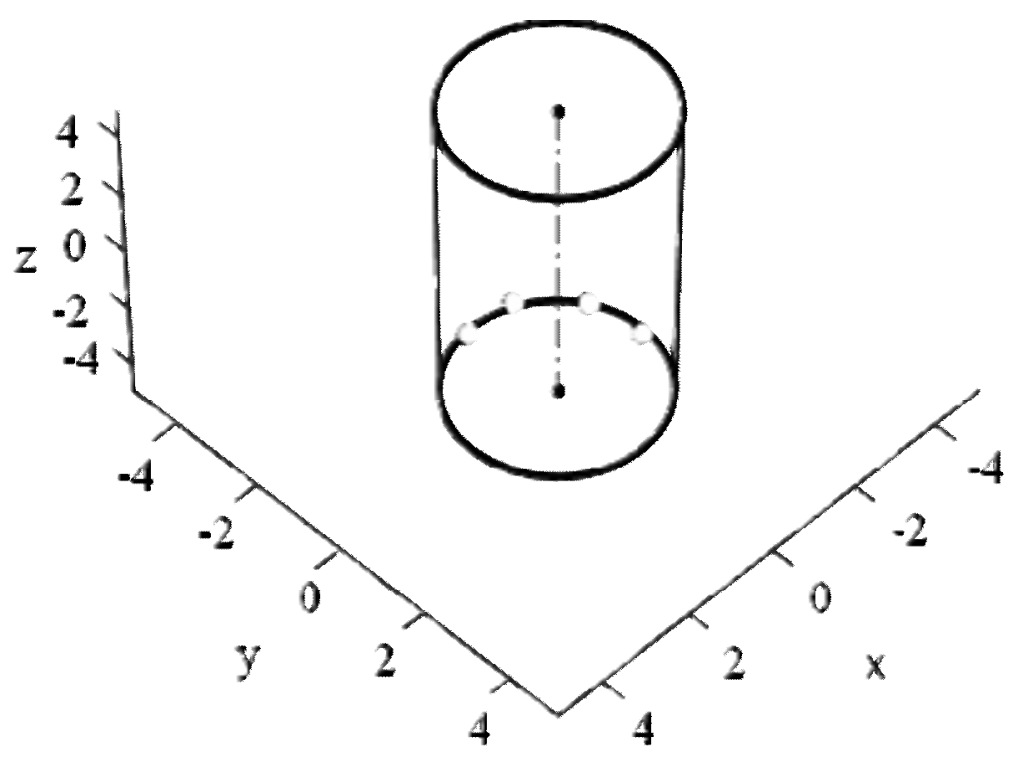

Figure (7.1): Demonstration of Test Case 1.

$$
\begin{array}{r}
11 \\
= \\
211 \\
-5 \\
-111 \\
-111
\end{array}
$$
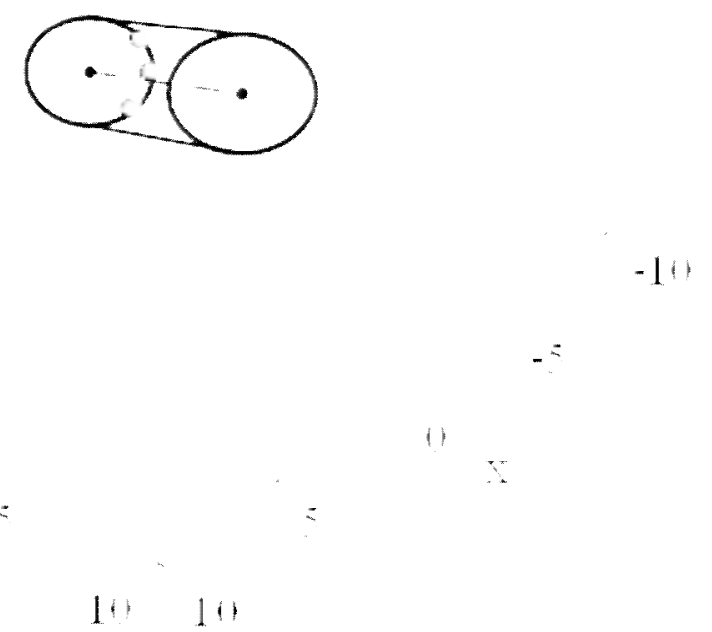

Figure (7.2): Demonstration of Test Case 2. 


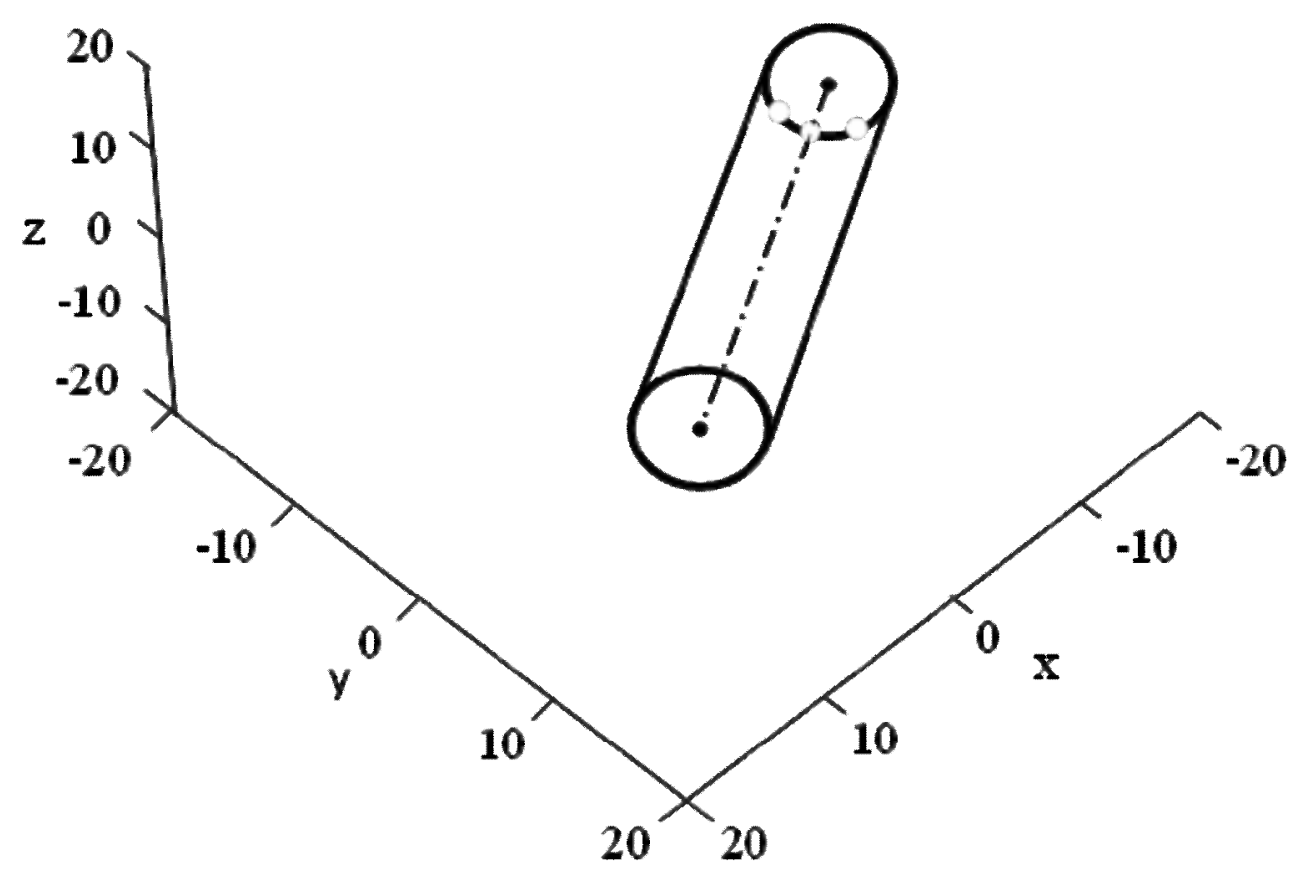

Figure (7.3): Demonstration of Test Case 3.

\section{CONCLUSION}

A right circular cylinder is one of the most important geometric primitives used in applications. In literature, cylinders are a subject of active research in many directions. Among them, computing cylinders from minimal sets of 3D-points, finding the smallest enclosing cylinders to a set of data points, cylindrical objects detection, recognition and extraction, and fitting a cylinder to a set of data points. In all this work, the geometric parameters of a cylinder play a central role.

The right circular cylinder is described by a set of parameters called the geometric parameters. These parameters are: a vector $\left(\lambda_{s} \mu_{s}, v\right)$ giving the direction of its axis, a point $\left(\alpha_{6}, y_{6}, z_{6}\right)$ to fix the axis-position, and a positive real number $R$ giving the radius of the cylinder. The algebraic equation of a right circular cylinder, as a member of quadric surfaces, is given. The coefficients of the algebraic equation are called the algebraic parameters. The algebraic parameters have no direct geometric meaning. The geometric equation of the RCC is given, in which the geometric parameters appear explicitly. The coefficients of the algebraic and geometric equations are compared resulting-in ten equations. The geometric parameters are expressed in terms of the algebraic parameters in three stages. In the first stage the components of the axis-direction vector is obtained after proving six propositions. In the second stage the coordinates of a point on the axis is obtained after proving a proposition and then solving three equations under some conditions. Three sets of solutions are obtained and only one of them is used according to the case considered. In the third stage the radius of the cylinder is obtained.

Finally, the proposed algorithm is introduced. The algorithm's input is the algebraic parameters while its output is the geometric parameters. A two-cycle loop is used to allow for using the algebraic parameters in the case of multiplying the RCC's algebraic equation by minus one. The possible spatial positions of a RCC are summarized in seven cases. Three cases when the axis is parallel to one of the principal axes, three cases when the axis is parallel to one of the principal planes, and a case when the axis is in general position. Each case appears in the algorithm through an IF-statement. The algorithm is tested on five cases including non-RCC cases. The results were exact. 


\section{REFERENCES}

1. Al-Subaihi, I.A., 2016], Geometric Fitting by two Coaxial Cylinders, Mathematical Communications, Vol. 21, pp. 227-237.

2. Beder, C. and Forstner, W., 2006], Direct Solutions for Computing Cylinders from Minimal Sets of 3D Points, In: Proceedings of European Conference on Computer Vision (ECCV2006), pp. 135-146.

3. Brandenberg, R. and Theobald, T., 2004], Algebraic Methods for Computing Smallest Enclosing and Circumscribing Cylinders of Simplices, Applicable Algebra in Engineering, Communication and Computing (AAECC), Vol. 14, No. 6, pp. 439-460.

4. Chaperon, T. and Goulette, F., 2001], Extracting cylinders in full 3D data using a random sampling method and the Gaussian image, In: Proceedings of Vision Modeling and Visualization Conference 2001 (VMV-01), pp. 35-42, Stuttgart, Germany.

5. Devillers, O. et al., 2001], On Circular Cylinders By Four Or Five Points In Space, Report No 4195, Institut National De Recherche En Informatique Et En Automatique, France.

6. Figueiredo, R. et al., 2017], Robust cylinder detection and pose estimation using 3D point cloud information, In: Proceedings of IEEE International Conference on Autonomous Robot Systems and Competitions (ICARSC),pp. 234-239, April 26-28, Coimbra, Portugal.

7. [Ilyin, V.A. and Poznyak, E.G., 1984], Analytic Geometry, pp. 191, Mir Publishers, Moscow.

8. Kwon, S. et al., 2003], Algorithms for fitting cylindrical objects to sparse range point clouds for rapid workspace modeling, In: Proceedings of $20^{\text {th }}$ International Symposium on Automation and Robotics in Construction, pp. 173-178.

9. Lichtblau, D., 2012], Cylinders Through Five Points: Computational Algebra and Geometry, Journal of Mathematics Research, Vol. 4, No. 6.

10. Lukacs, G. et al., 1998], Faithful Least-Squares Fitting of Spheres, Cylinders, Cones

11. and Tori for Reliable Segmentation, In: Proceedings of 5th European Conference on

12. Computer Vision (ECCV'98), pp. 671-686, Freiburg, Germany, June 2-6.

13. Mortenson, M.E., 1990], Computer Graphics Handbook: Geometry and Mathematics, pp. 167, Industrial Press Inc., New York.

14. Nurunnabi, A. et al., 2017], Robust cylinder fitting in three-dimensional point cloud data, International Archives of the Photogrammetry, Remote Sensing and Spatial Information Sciences, Volume XLII-1/W1, pp. 63-70.

15. Pogorelov, A.V., 1980], Analytic Geometry, pp. 154, Mir Publishers, Moscow.

16. Rabbani, T. and Heuvel, F., 2005], Efficient Hough Transform for Automatic Detection of Cylinders in Point Clouds, In: Proceedings of ISPRS WG III/3, III/4, V/3 Workshop "Laser scanning 2005", Enschede, pp. 60-65, the Netherlands, September 12-14.

17. Sarcar, M. et al., 2014], Automatic Feature Recognition of Cylinder and Knuckle Thread from Neutral Files, 5th International \& 26th All India Manufacturing Technology, In: Proceedings of Design and Research Conference (AIMTDR 2014), December 12th-14th, IIT Guwahati, Assam, India.

18. Schomer, E. et al., 1996], Smallest Enclosing Cylinders, In: Proceedings of the twelfth annual symposium on computational geometry.

19. Toony, Z. et al., 2015], PGP2X: Principal Geometric Primitives Parameters Extraction, In: Proceedings of the $10^{\text {th }}$ International Joint Conference on Computer Vision, Imaging and Computer Graphics Theory and Applications (VISIGRAPP2015), Berlin, Germany.

20. Tran, T. et al., 2015], Extraction of cylinders and estimation of their parameters from point clouds, Computers \& Graphics, Vol. 46, pp. 345-357.

21. Watson, G.A., 2006], Fitting enclosing cylinders to data in $\mathrm{R}^{\mathrm{n}}$, Numerical Algorithms, Vol. 43, No. 2, pp. 189-196. 